\title{
Ciudad intrusa y ciudad simbionte: Desde la autosuficiencia a la ecología urbana
}

\author{
Nieves Mestre Martínez \\ Departamento de Expresión Gráfica y Proyectos Arquitectónicos \\ Escuela de Arquitectura \\ Universidad Europea de Madrid \\ nieves.mestre@uem.es
}

Recibido: $11 / 09 / 2014$

Modificado: $30 / 10 / 2014$

Aceptado: $22 / 11 / 2014$

\section{Resumen}

Contemporáneamente al nacimiento del rascacielos, el edificio híbrido se vincula a una práctica experimental y en cierto modo disidente de la disciplina. Los volúmenes puros de la Modernidad serán sometidos a una diagnosis crítica: primero de orden programático -mixed-use-, luego estructural y por último ambiental y ecológica. Estas revisiones, en especial la ecológica, comprometerán también la definición de su contexto urbano, proponiendo con ello modelos de ciudad alternativos. Algunos de ellos anticipan la agenda que centrará la producción arquitectónica y urbana de la contemporaneidad más comprometida.

Palabras clave: edificio híbrido, ecología urbana, autosuficiencia, urbanismo radical.

Title: Intrude City and Simbiont City. From Self-sufficiency towards Urban Ecology

\section{Abstract}

Contemporarily to the birth of skyscrapers, hybrid building gets linked to an experimental practice and somehow dissident towards discipline. Pure volumes of modernity will be submitted to a critical diagnosis: firstly concerning the program -mixed-use- then the structure, and later on the environment. These revisions, especially the latter, will compromise the definition of its immediate urban context, proposing alternative city models. Some of them get to anticipate the agenda that will center the urban and architectural production of Contemporaneity.

Keywords: hybrid building, urban ecology, self-sufficiency, radical urbanism.

\section{Índice}

1. Introducción: Revisiones mecánicas y aerodinámicas del modelo en altura

2. Ciudades intrusas: Habitar otros medios

3. Parásitos y simbiontes. Arquitectura inquilina

4. Urbanismo bajo en emisiones 


\section{Introducción: Revisiones mecánicas y aerodinámicas del modelo en altura}

Así como la reconsideración funcional del rascacielos moderno fomentó el nacimiento de mixed-use a principios del siglo $\mathrm{XX}$, en el periodo de entreguerras se sucedieron una serie de revisiones del modelo vertical en términos estructurales. Tanto las propuestas de la vanguardia constructivista como los ensayos aerodinámicos de Buckminster Fuller diagnosticaron prematuramente la "ineficacia estructural" del prisma moderno (Sentkiewicz 2011: 32). Estas experiencias detectaron asimismo los beneficios estructurales derivados de la especialización del cálculo, dando lugar a configuraciones mestizas y no prismáticas.

La verticalidad del rascacielos se desafió en casi todos los modelos en altura propuestos por el constructivismo ruso: desde las pruebas de máxima esbeltez de Ian Leonidov en el Lenin Institute (1927), hasta las propuestas diagonales y anti-gravitatorias de El Lissitzky, Vladimir Tatlin o Georgii Krutikov ${ }^{1}$. Desde una óptica menos poética, Buckminster Fuller aborda en la misma fecha los requisitos de la aerodinámica pasiva en el carenado del modelo 4D Timelock. La propuesta consigue "economizar energía, tanto calorífica como estructural" respecto al prisma moderno (Ábalos y Herreros 1992: 89) y fue aplicada años más tarde a escala urbana en su propuesta Dome over Manhattan. Esta cúpula de $3 \mathrm{~km}$ de diámetro pretendía paliar la ineficacia ambiental de la ciudad, superponiendo una forma térmica antitética sobre su skyline denticulado².

A partir de la segunda mitad de siglo, estas revisiones mecánicas y aerodinámicas dejan paso a la consideración ambiental del modelo. El precepto mecánico se sustituye por el ideal orgánico, y la naturaleza se introduce como presupuesto ineludible. Sobre esta premisa se suceden diversas formas de hibridación natural-artificial: desde los más literales ensayos de biomímesis hasta propuestas más sofisticadas de reconstrucción ambiental. Casi todos los arquitectos de la Posmodernidad ${ }^{3}$ defendieron entonces "su compromiso con lo híbrido o el espacio intersticial" (Jencks 2002: 210).

Esta mezcla estratégica de ciudad y naturaleza había sido anticipada por el Estilo Internacional mediante calculados encuadres y poca rentabilidad funcional. Frente al paisaje inerte sobre el que

1 Su propuesta de Ciudad Flotante puede considerarse una de las más radicales (vid. Salter 2010).

${ }^{2}$ El propio Fuller lo comparaba con las aletas de refrigeración de un motor. Esta configuración, muy deseable en verano, difícilmente podría resultar más ineficaz para la estación invernal (vid. Steadman 1979).

3 El término "posmoderno" se refiere en el texto al periodo entre 1957, año del último Congreso del CIAM, y 1982, año del concurso de La Villette. Con esta demarcación pretende ampliarse la habitual identificación del término con la Posmodernidad neo-historicista de los años 70 y 80. 
gravita la Casa Farnsworth, el paisaje en la Posmodernidad pasa a ser objeto de transformación artificial. La naturaleza deja de ser un fondo neutro sobre el que se destaca el objeto arquitectónico, para entablar con él una interlocución creciente. La manufactura Posmoderna conquista sucesivamente la producción y reproducción ${ }^{4}$ de la naturaleza por medios artificiales. La entidad vertical, ya distorsionada por la Modernidad disidente, vuelve a revisarse al amparo de una incipiente cultura tecno-ecológica.

En este contexto se propone la lectura contrastada entre dos modelos coetáneos: los ensayos de ciudades intrusas en contextos naturales extremos y los producidos como prótesis de naturaleza en entornos urbanos consolidados. Los primeros surgieron de la colaboración interdisciplinar del programa de la NASA a partir de 1961 , y sus conclusiones fueron muy nutritivas para la arquitectura en la definición del hábitat autosuficiente; los segundos se desarrollaron con especial intensidad en los colectivos experimentales austriacos, centrados en torno a la generación de atmósferas artificiales por medios tecnológicos. Estos últimos se caracterizan por la ocupación de entornos urbanos consolidados, sobre los que la naturaleza encapsulada ejerce una acción paliativa y rehabilitadora.

\section{Ciudades intrusas: Habitar otros medios}

El ambicioso programa aeroespacial anunciado por Kennedy en 1961 animó a la Ecological Society a lanzar una serie de conferencias sobre ecología espacial para establecer alianzas entre ecólogos, investigadores espaciales e ingenieros militares. Estas tuvieron lugar entre 1963 y 1965 en la Universidad de Princeton, en colaboración con la recién fundada NASA, bajo el título de "Human Ecology in Space Flight". Los hermanos Eugene y Howard T. Odum hicieron importantes aportaciones, aplicando la Teoría de los Sistemas Energéticos a la ecología.

Toda una generación de arquitectos empieza en este momento a descubrir la capacidad real de la tecnología para generar atmósferas domesticadas y artificiales $^{5}$. Pero los verdaderos precursores de este cambio fueron Cedric Price y Buckminster Fuller, a los que unía un interés común por la ecología y la cibernética. Ante una inminente crisis energética y bajo la constante amenaza de una guerra nuclear, Fuller imparte en 1964 una de sus más famosas conferencias: "Spaceship Earth". En ella proponía la traslación de los

${ }^{4}$ El concepto de reconstrucción natural se aborda de forma extensiva en Gissen (2009).

${ }^{5}$ El propio Le Corbusier puede considerarse pionero en la aplicación arquitectónica de la ecología de la cápsula espacial. En el mur neutralisant describe "referencias numéricas muy precisas de temperatura y humedad" premonitorias (vid. Banham 1967). 
sistemas de gerencia de las estructuras aeroespaciales para la gestión ecológica de la arquitectura y de nuestro planeta (Anker 2005). Con distintos patrocinios, el discurso de la autosuficiencia siguió alentando su obra ${ }^{6}$ durante las siguientes décadas, dando pie a la Ilamada Cabin Ecology ${ }^{7}$. Su influencia será notable en toda una saga de diseñadores y arquitectos en pos del hábitat autónomo (Anker 2005).

En 1967, Architectural Design publica un número especial titulado "2000+" comisariado por John McHale, por entonces director del Fuller's World Resources Inventory ${ }^{8}$. El texto reunía un buen número de cápsulas, estaciones espaciales y vehículos subacuáticos: ecosistemas de ciclo cerrado que anticipaban muchas de las soluciones de hábitat autosuficiente que proliferaron en la década siguiente. La unión entre ecología, cibernética y tecnología submarina sería, según McHale, la revolución más notable de la humanidad (Anker 2005).

Esta contaminación disciplinar se consolida a finales de los 60 como corriente arquitectónica: acuñada por el alemán Rudolph Doernach en 1966, la biotectura nace como un híbrido entre biología y arquitectura. Fundador del Instituto de Biotectura de Güllingen, Doernach desarrolló modelos de ciudades iceberg, ciudades comestibles y ciudades flotantes. En ellas, la única acometida energética disponible procede del propio sustrato natural, ya sea el océano, el desierto o el hielo. Tanto Hidrópolis (1966) como Iceland (1964) se diseñan intuitivamente sobre la base del excedente energético, pero su viabilidad no consigue explicitarse. Sus presupuestos, igual que los del metabolismo, aludían a un intercambio de materia y energía con el medio físico para el que la arquitectura no tenía todavía capacidad tecnológica ${ }^{9}$.

Conceptos híbridos similares al de Doernach proliferaron en estos años. Las Arcologías ${ }^{10}$ de Paolo Soleri establecían vínculos entre arquitectura y ecología, y suponen por ende una contribución fundamental al discurso sobre autosuficiencia. Las ciudades de Soleri son laboratorios habitables que apuestan por la autosuficiencia y la

${ }^{6}$ En proyectos como Climatroffice, diseñado en 1971 junto a un joven Norman Foster (vid. Treiber 1995).

7 Término empleado por la ingeniería aeroespacial para designar el ambiente en el interior de un vehículo espacial. En él se reproducía por medios mecánicos la naturaleza de la biosfera, incluyendo el movimiento de aire, agua y nutrientes (Anker 2005).

${ }^{8}$ Será él quien investigue los conceptos energéticos y ecológicos implícitos en los primeros prototipos de Fuller, así como su explicación científica (vid. McHale 1969).

${ }^{9}$ Revisiones posteriores, como Ciudad Iceberg (1975) de José María Prada Poole, verificarán su viabilidad energética.

${ }^{10}$ El término resulta de la cooperación sintáctica entre arquitectura y ecología (vid. Soleri 1969). 
densidad vertical: viviendas, oficinas, escuelas y centros culturales se integran con producción alimentaria y energética en un diseño urbano hiperorgánico (Grierson 2007). Las mejoras mecánicas y estructurales del rascacielos comienzan entonces a combinarse con un interés por su "potencial termodinámico" (Sentkiewicz 2011: 32).

\section{Parásitos y simbiontes. Arquitectura Inquilina ${ }^{11}$}

Aunque las infraestructuras del Megaestructuralismo y Metabolismo se diseñaron con capacidad de crecimiento orgánico, la reflexión ambiental del urbanismo era hasta ese momento un tema poco explorado. Su rango temporal se emplaza a uno y otro lado de la crisis del petróleo, asumiendo un compromiso cuestionable con la ecología. Stus esquemas urbanos se demos $\downarrow$ isaron pronto inadecuados , y fueron sustituidos a partir de 1964 por acciones protésicas y puntuales sobre la ciudad existente. Frente a los escenarios utópicos y las visiones cenitales de los primeros, empezaron a proliferar las reflexiones efectivas sobre la ciudad real y su entorno concreto.

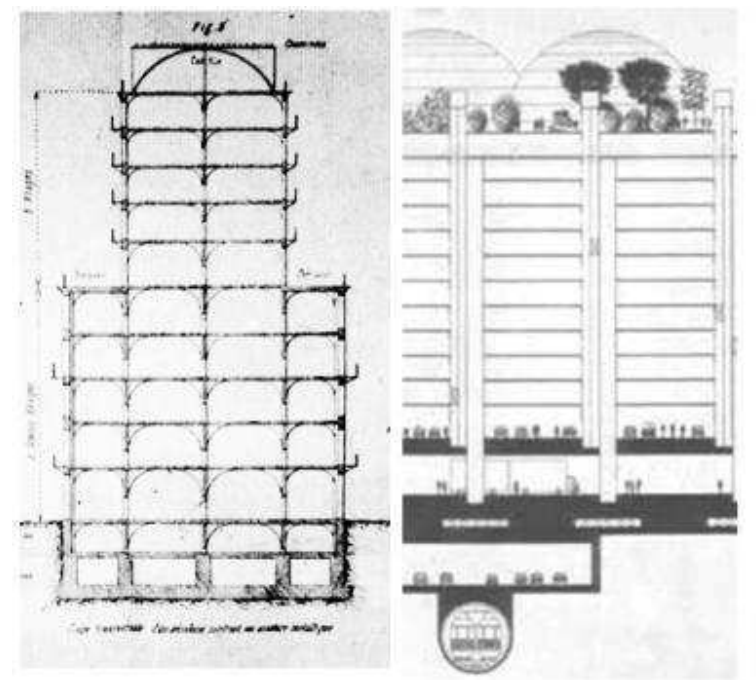

Figura 1. Aerodomes, de J. Borie (1867); No-stop city, de Archizoom (1968); Instant City, de Archigram (1969).

${ }^{11}$ El término se toma de las Estructuras de Ocupación de Willy Muller, "adaptadas o adheridas a las superficies vacías de la ciudad" (Muller 2000).

${ }^{12} \mathrm{Ni}$ la ciudad contemporánea ni la tradicional pueden explicarse con estructuras modulares sino a través de estructuras solapadas y complejas (vid. Alexander 1966).

${ }^{13}$ Año que coincide con el estallido de las estructuras neumáticas (Dessauce 1999). 
Los beneficios de la acupuntura urbana ${ }^{14}$ no son exclusivos de la ciudad contemporánea, con precedentes europeos de más de un siglo de antigüedad. Henri Jules Borie planteaba ya en 1867 una solución regeneradora para los suburbios insalubres de la periferia parisina (figura 1). Los Aerodomes congregan una serie de pequeñas construcciones sobre las cubiertas infrautilizadas de edificios de viviendas, destinados a dar cabida a la creciente demanda de usos públicos. Definidas implícitamente en los objetos-tipo del primer Le Corbusier ${ }^{15}$, estas prótesis irán sofisticándose hasta facilitar el hábitat transportable del universo Archigram ${ }^{16}$. Tanto Tuned Subsurbs como Instant City (1968 y 1969), proponen la ocupación explícita de arquitecturas preexistentes, sobre las que una serie de artefactos ejercen una rehabilitación tecnológica ${ }^{17}$. Aunque sus beneficios se definen en términos funcionales ${ }^{18}$, estas acciones parecen advertir ya su capacidad terapéutica ${ }^{19}$ (Vidler 1992). La metáfora del parásito reúne las condiciones de "adyacencia (del latín pará) y alimento (del latín síto)" (Marini 2008: 111), e indica por tanto una dependencia infraestructural de las intervenciones sobre la preexistencia.
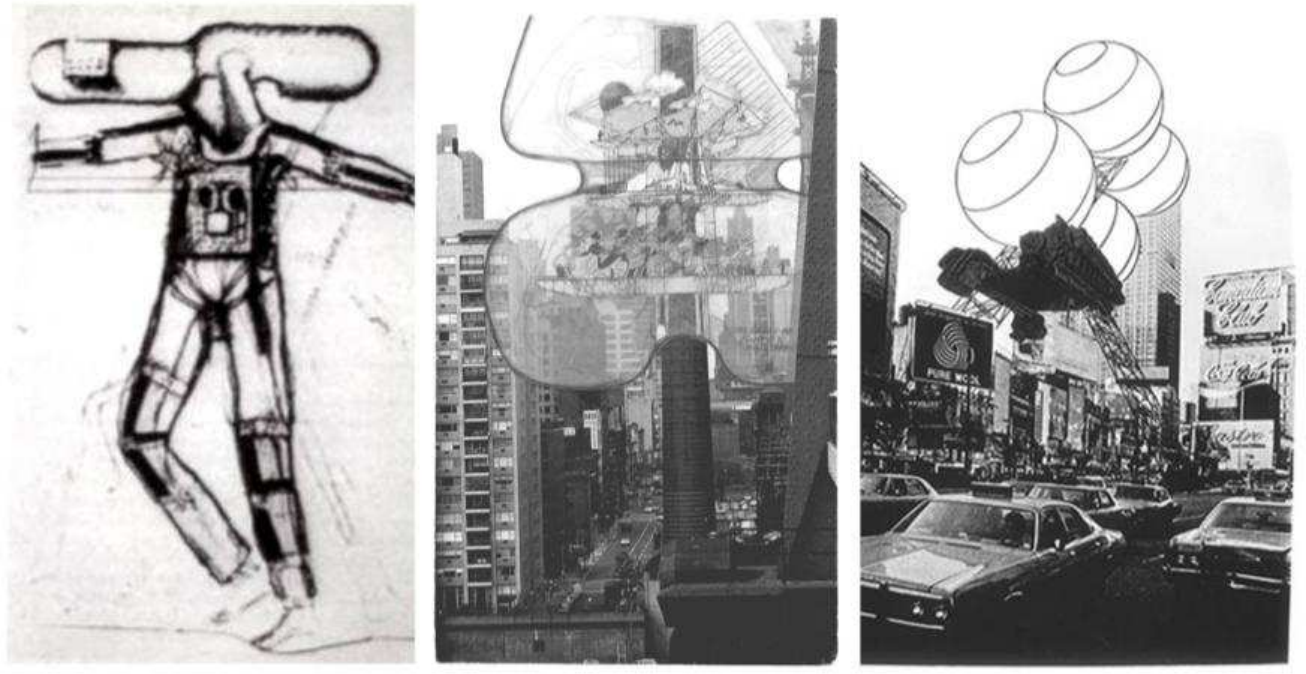

Figura 2. Stándar Suite, de Walter Pichler (1963); Air Spa Hotel (1970) y Pneumacosmic Formation (1971), de Haus-Rucker-Co.

\footnotetext{
${ }^{14}$ Término tomado de Solà-Morales (2004).

15 El ambiente controlado y diferenciado de los object-types en el continuum doméstico, permite actividades del cuerpo humano diferenciadas del resto de la casa (Vidler 1992).

${ }_{16}$ Propuestas como la Drive-in House (1964), Cushicle (1966) o Suitaloon (1976).

17 Con dicha rehabilitación, Archigram demuestra que los habitantes de los suburbios podrían tener experiencias tan intensas como los de los centros metropolitanos (vid. Jong y Schuilenburg 2006).

18 Service Tower (Londres, 1967) de Grimshaw y Farrell puede considerarse un ejemplo paradigmático de "prótesis funcional" engarzada en un edificio del siglo XIX (Sadler 2005: 109).

${ }^{19}$ El nuevo hábitat era a un tiempo "prótesis y profilaxis" (Vidler 1992: 147).
} 
La producción de la vanguardia vienesa entre los años 60 y 70 representa una casuística muy particular. Definida por propuestas dirigidas a aumentar la movilidad del ocupante y "la recreación medioambiental del espacio", su actividad fue extensamente analizada en su momento (Cook 1970: 74; Burns 1972: 100). Pese a su elevada disparidad, los textos permiten establecer una preferencia generalizada por la tecnología neumática y el carácter protésico de sus intervenciones, llegando a identificarse como "fenómeno austriaco" (Cook 1970).

La vanguardia austriaca (cfr. Porsch 2009) supo reunir las referencias del viaje aeroespacial con las de la primera revolución mediática. Sobre los presupuestos de transportabilidad de Archigram importaron los de mediación climática, muy visibles en propuestas como Bioadapter y Standar Suite, diseñadas por Walter Pichler en 1967 (figura 2). Las propuestas coetáneas de Haus-Rücker-Co priorizaron la caracterización energética sobre la digital, especialmente en Pneumacosm. A su zaga, numerosos enunciados ${ }^{20}$ posteriores se basaron en estructuras urbanas para equipar temporalmente determinadas zonas de la ciudad de Viena. Frente a los Oasis $^{21}$ de Archigram, los de Rucker se sitúan ya en la crisis del petróleo y persiguen una cooperación ambiental recíproca. En lugar de usurpar la energía del huésped, permiten su regeneración ambiental y funcional, con estructuras climatizadas que albergan jardines o programas de ocio (Martínez 2005).

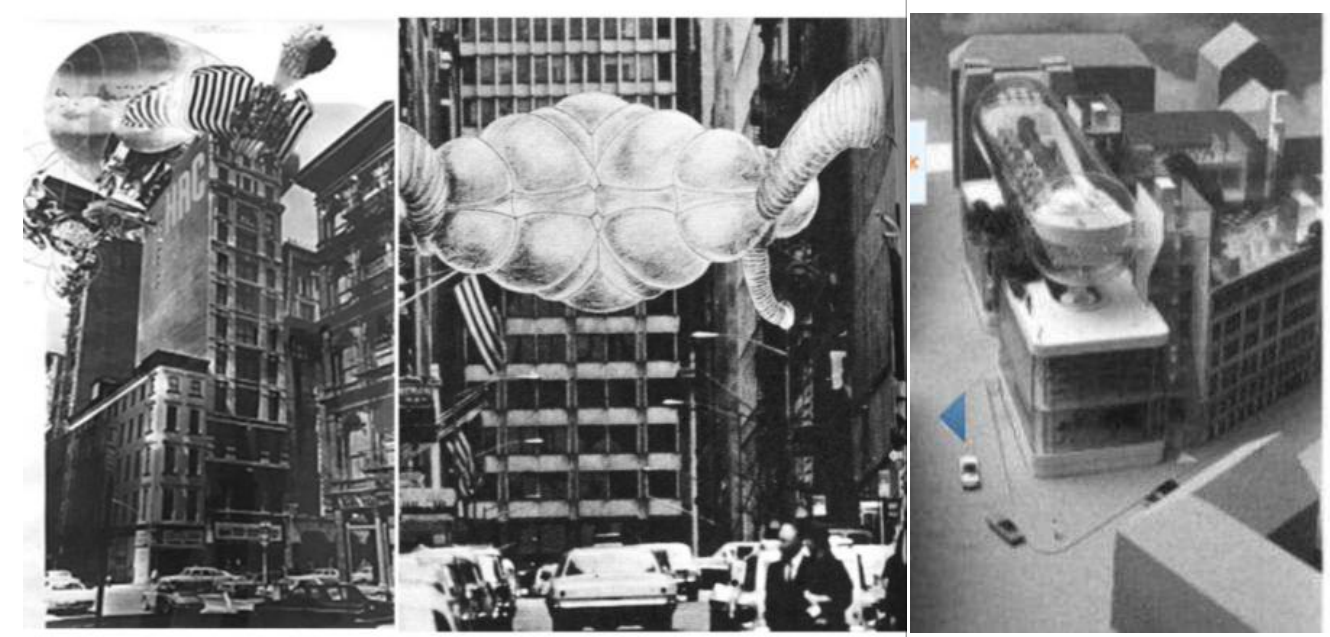

Figura 3. Rooftop Oasis (1976), de Haus- Rucker-Co; Children Cloud (1967), de Missing Link; Frischzelle, de Coop Himmelblau.

20 Otros grupos también nacidos en la Viena de los 60, como Missing Link Productions (con Angela Hareiter), presentan numerosos proyectos de vocación parásita dentro de la ciudad. Golden Viennese Heart o SIA CON ALT son propuestas del grupo de 1970.

${ }^{21}$ Oasis es una propuesta de Ron Herron en 1968, de la que quizá los Rooftop Oasis de Rucker tomen su nombre (Thomsen 1994). 
Simultáneas a las de Rucker, las experiencias de CoopHimmelblau insistieron en la colonización de estructuras preexistentes. La cooperativa sustituyó el concepto de "oasis" por el de "nube"22, una fructífera combinación de tecnología neumática y movilidad mecánica desarrollada con insistencia entre 1968 y 1972. A partir de entonces, comenzó a recibir encargos reales de rehabilitación para cubiertas tipo mansarda en el centro histórico de Viena. Frischzelle (figura 3) se concretó en un gran conducto de vidrio extendido a lo largo de varias cubiertas adyacentes. En su interior se proyectó un parque bioclimático vertical definido con autonomía energética y con capacidad de regeneración sobre el edificio anfitrión. La componente geométrica de sus propuestas posteriores se alinea con la agenda del deconstructivismo (Thomsen 1994), abandonando en cierta medida sus compromisos iniciales.

\section{Urbanismo bajo en emisiones}

El símil biológico ha sido una constante en las agendas de la arquitectura y el urbanismo desde el embargo energético de los 70. $\mathrm{Si}$ los primeros ejemplos establecen las bases para el diseño eficiente, los segundos habilitan las bases del urbanismo ecológico. EI símil del ecosistema urbano requiere el solape de ambas: el procesado de residuos es necesario para neutralizar la huella de carbono asociada a los procesos de autosuficiencia energética (Bermejo 2005: 258).

En Occidente las ordenanzas han sancionado la superposición de usos y tipologías diversos con un diseño urbano heredero del funcionalismo moderno. Así se ha evitado la aparición de supuestos efectos negativos sobre la habitabilidad del entorno construido: sombras arrojadas, emisión acústica o lumínica, servidumbres de acceso, emisión de residuos, e incluso discordancias estéticas. Pero estas prescripciones también han reprimido un buen número de colindancias que podrían considerarse como beneficiosas en términos energéticos: amortización de excedentes térmicos, aprovechamiento de inercia térmica o estructural de una entidad sobre otra, apantallamiento acústico, protección solar o aerodinámica, etc.

Pese a este absentismo histórico, recientes ordenanzas en Norteamérica y Norte de Europa empiezan a favorecer el agregado multifuncional por sus probados beneficios sobre la salud urbana (Marini 2008). La emisión de normas urbanísticas para restringir la nueva edificación y transformar la existente en los centros urbanos, ha llevado el debate urbano de muchas capitales europeas hacia el reciclaje y la reutilización. A diferencia de las occidentales, las

${ }^{22}$ El concepto está también en este caso tomado de diseños previos de Archigram. Cloud puede vincularse con el Blow Out Village, diseñado por Peter Cook en 1966 (vid. Hejduk 2006). 
ordenanzas japonesas no están reguladas por usos sino por intensidades, y permitirán el arraigo de una singular forma de hibridación urbana ${ }^{23}$.

La adecuada catalogación de los excedentes energéticos permitirá avanzar hacia un urbanismo bajo en emisiones (Van den Dobbelsteen 2010). Uno de los requerimientos esenciales sería establecer precisamente las relaciones significativas entre ámbitos demandantes y disipadores de energía en el conjunto de la ciudad y detectar usos compatibles. Esto requeriría de "un nuevo tipo de infraestructura, que operaría a menor escala que nuestras actuales redes energéticas" (Van den Dobbelsteen 2010: 268). Este concepto ha sido abordado de forma explícita por el estudio 2012 Architecten a través de una investigación multidisciplinar: esta se caracteriza por reconsiderar las redes de procesos y flujos existentes, facilitando "nuevas conexiones entre exceso y demanda existentes a nivel local" (Jongert, Nelson y Goosens 2011: 56). El sistema (vid. 2012 Architecten 2014) actúa como un procesador metabólico, invirtiendo con ello la tendencia urbana a dispersar entrópicamente materiales y energía.

\section{Bibliografía}

2012 Architecten (2014): Cyclifier. Inventive Ways to optimally use our urban resources. En: http://www.cyclifier.org [Consulta: 30/10/2014].

ÁBALOS, Iñaki; y HERREROS, Juan (1992): Técnica y Arquitectura en la ciudad contemporánea: 1950-2000. Guipúzcoa: Nerea.

ALEXANDER, Christopher (1966): "A city is not a tree". Design, núm. 206, February 1966, pp. 46-55.

ANKER, Peder (2005): "The ecological colonization of space". Environmental History, vol. 10, núm. 2, pp. 239-268.

BANHAM, Reyner (1967): The Architecture of the Well Tempered Environment. Londres: The Architectural Press.

BERMEJO, Roberto (2005): La gran transición hacia la sostenibilidad. Principios y estrategias de economía sostenible. Madrid: Catarata.

BURNS, John (1972): Arthropods: New Design Futures. Nueva York: Praeger.

COOK, Peter (1970): Experimental Architecture. Nueva York: Universe Books.

DESSAUCE, Marc (1999): The inflatable moment: Pneumatics and Protest in '68. Princeton: Princeton Architectural Press.

${ }^{23}$ Made in Tokio (Kuroda y Kaijima, 2001) registra casos que vinculan categorías tan diversas como paisaje, infraestructura, arquitectura o mobiliario urbano. La mayor divergencia entre dichas categorías redunda en un mayor aprovechamiento ecológico: los entornos resultantes se denominan no por casualidad "unidades ambientales" (Marini 2008: 11-12). 
GISSEN, David (2009): "The Architectural Production of Nature, Dendur/New York". Grey Room, núm. 34, Winter, pp. 58-79.

GRIERSON, David (2007): "Arcology and Arcosanti. Towards a sustainable built environment". Global Environment. Problems and Policies, vol. 1 , pp. 194-213.

HEJDUK, Renata (2006): "A Generation on the Move. The Emancipator Function of Architecture in the Radical Avant Garde 1960-1972", en Robert Kronenburg y Filiz Klassen (eds.), Transportable Environments, pp. 40-52. Oxon: Taylor and Francis.

JENCKS, Charles (2002): The New Paradigm in Architecture. The Language of Posmodernism. Londres / New Haven: Yale University Press.

JONG, Alex; y SCHUILENBURG, Marc (2006): Mediapolis: Popular Culture and the City. Rotterdam: 010 Publishers.

JONGERT, Jan; NELSON, Nels; y GOOSENS, Fabienne (2011): "Recyclicity. A Tool for Resource-based Design". Architectural Design. núm. 214, pp. 54-62.

MARINI, Sara (2008): Parasite Architecture. Recycling Strategies for the Contemporary City. Macerata: Quodlibet.

MARTÍNEZ, Andrés (2005): Habitar la cubierta. Barcelona: Gustavo Gili.

McHALE, John (1969): "An Ecological Overview", en The Future of the Future, pp. 66-74. New York: George Braziller.

MULLER, Willy (2000): "Ad ARch, Estructuras de Ocupación". Fisuras, núm. 8, Enero.

PORSCH, Johannes (2009): Architektur Avangarde Österreich: 1956-1973. Berlín: Birkhäuser.

SADLER, Simon (2005): Archigram: Architecture without architecture. Massachusets: MIT Press.

SALTER, Chris (2010): Entangled, Technology and the Transformation of Performance. Massachusetts: MIT Press.

SENTKIEWICZ, Renata (2011): "Verticalscapes: Concepto y caracteres", en Campos prototipológicos termodinámicos. Colección de Textos académicos ETSAM-UPM, vol. III, pp. 29-35.

SOLÀ-MORALES, Manuel de (2004): "The strategy of urban acupuncture". Structure Fabric and Topography Conference, Nanjing University.

SOLERI, Paolo (1969): Arcology: The City in the Image of Man. Arizona: Cosanti Press.

STEADMAN, Philip (1979): The evolution of Design. Biological Analogy in Architecture and the Applied Arts. Londres: Routledge.

THOMSEN, Christian (1994): "Mediarchitecture. The Viennese Avantgarde of the 60's and beyond". Architecture and Urbanism, vol. 289, pp. 7683.

TREIBER, Daniel (1995): Norman Foster. Londres: E \& FN Spon.

VAN DEN DOBBELSTEEN, Andy (2010): "Synergy, Not Autarky", en The Why Factory, Winy Maas, Pirjo Haikola y Ulf Hackauf (eds.), Green Dream. How Cities Can Outsmart Nature, pp. 266-270. Rotterdam: The Why Factory / Nai Publishers.

VIDLER, Anthony (1992): "Homes for Cyborgs", en Anthony Vidler (ed.), The Architectural Uncanny: Essays in the Modern Unhomely. Massachussets: MIT Press. 